\title{
Propagation of stress fracture of the patella
}

\section{A A Crowther, A Mandal, P P Sarangi}

Br J Sports Med 2005;39:e6 (http://www.bjsportmed.com/cgi/content/full/39/2/e6). doi: 10.1136/bjsm.2003.010116

Anterior knee pain in athletes is common and usually self limited. There should be a high index of suspicion and low threshold for special imaging in cases with acute onset and specific tenderness. The risk of propagation of stress fracture of the patella in athletes is highlighted. The case report presented illustrates the potential sequence of events.

$\mathrm{S}$ tress fractures commonly occur in distance runners, military recruits, and patients with cerebral palsy with knee flexion contractures. ${ }^{1}$ Exposures to forces applied in a rhythmic, repetitive, and sub-threshold manner can cause this form of overuse injury, but even in these groups stress fracture of the patella is rare. Such injury is recognised in patients who have undergone bone-patellar tendon-bone anterior cruciate ligament reconstructions and those with patella resurfacing in total knee arthroplasty. Stress fractures are generally classified according to cause, which is either fatigue or insufficiency. ${ }^{2}$ Fatigue fractures are either complete or incomplete and occur in healthy bone as a result of submaximal stress loads. Insufficiency fractures occur in a previously weakened bone under physiological stress. Here we describe a case of stress fracture of the superficial surface of the patella progressing to complete transverse fracture.

\section{CASE REPORT}

A 35 year old man experienced acute left knee pain while serving during tennis. He is a sedentary worker with no comorbidities who enjoys competitive social tennis $2-3$ times a week. He denied a recent increase in activity and had experienced no other local trauma. The initial symptoms settled with oral analgesics and physiotherapy, but he presented three months later with persistent anterior knee

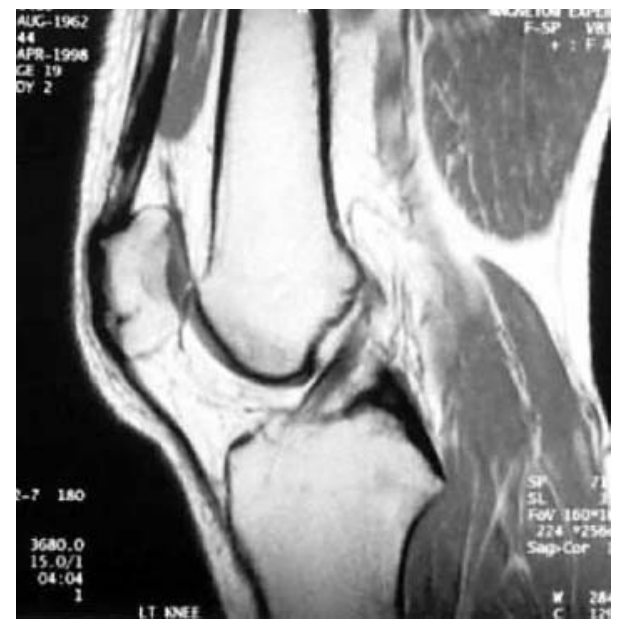

Figure 1 Incomplete transverse fracture of the patella extending from the anterior cortex. pain aggravated by physical activity, which was limiting his leisure pursuits. On examination, there was localised tenderness over the patella, but a full range of motion and no joint laxity. Plain radiographs were normal. Because of the chronic history and specific examination finding of localised tenderness, a magnetic resonance imaging scan was requested. This further investigation revealed an incomplete transverse fracture of the patella extending from the anterior cortex (fig 1). The patient was counselled about his condition, but he declined the prophylactic surgical treatment offered. Approximately two weeks later, while he was descending a step, his knee gave way as a result of a completely displaced transverse fracture of the patella (fig 2). He underwent surgery, and the fracture was internally fixed by a tension band technique. Ten months after surgery, he had returned to his previous level of activity with no residual symptoms.

\section{DISCUSSION}

Anterior knee pain in athletes is common. It is usually gradual in onset and settles with rest, analgesics, and/or antiinflammatory preparations and physiotherapy.

Stress fractures in athletes are most commonly seen in the tibia $(50 \%)$ and tarsal bones $(25 \%)$ but are recognised in other skeletal sites such as metatarsals $(9 \%)$, femur $(7 \%)$, fibula $(6 \%)$, pelvis $(1.5 \%)$, sesamoids $(0.9 \%)$, and spine $(0.6 \%)^{2}$

During normal activity the anterior patella is exposed to tensile stress while compressive forces are transmitted to the subchondral zone. High repetitions of loads below the yield strength of a material may result in stress fracture. Stress fractures of the patella are probably caused by a combination of muscular weakness, which reduces shock absorption, and

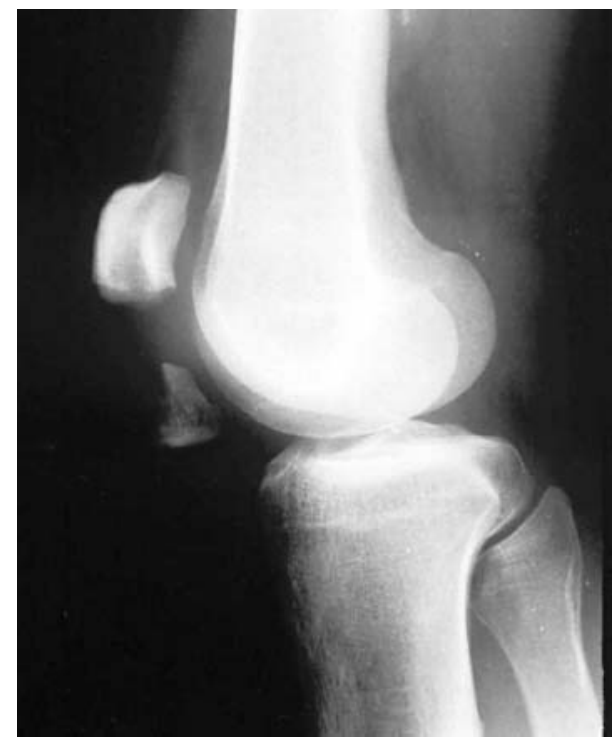

Figure 2 Completely displaced transverse fracture of the patella. 


\section{What is already known on the topic}

Stress fractures of the patella are rare but well documented in sportspersons. It has been recognised that undisplaced fractures of any bone may displace and that any stress fracture may propagate, complete, and displace.

\section{What this paper adds}

We alert the readership to the possibility of an undisplaced stress fracture of the patella in a sportsperson with anterior knee pain, a tender patella but normal plain radiographs. We illustrate the need for magnetic resonance imaging to confirm the diagnosis in such an instance. This case also shows the potential deterioration and outcome of fracture propagation and displacement if prophylactic internal fixation is declined.

muscle contraction, which initiates a disturbance in bone remodelling. ${ }^{3}$ Normally, remodelling is required to prevent failure in a bone subjected to repetitive bending moments.

Radiological diagnosis of stress fractures is difficult; initial radiographs will be sensitive in only a third of the cases. More accurate and sensitive investigation tools are bone scan or magnetic resonance imaging. It is difficult to make definitive treatment recommendations for the patella stress fracture because of the small number of reported series. However, as seen in this case, untreated or misdiagnosed stress fractures of the patella may develop into complete fractures, and we would recommend further radiological investigation in an athlete with a history of acute onset anterior knee pain, localised tenderness on clinical examination, and normal plain radiographs.

Once a stress fracture is evident, treatment should be tailored to suit the individual patient. Stress fracture on the tensile superficial surface of the patella is at risk of proceeding to complete fracture. Non-operative treatment with careful observation may be the right option for those patients who do not require immediate return to activity, ${ }^{4}$ but, for patients involved in high demand sports such as soccer, tennis, basketball, or weightlifting, ${ }^{5}$ prophylactic internal fixation using a standard tension band wiring technique is appropriate. Prognosis appears to be favourable, as full recovery has been seen in previously reported cases. ${ }^{4}$

\section{Authors' affiliations}

M A A Crowther, A Mandal, P P Sarangi, Bristol Royal Infirmary, Bristol, UK

Competing interests: none declared

Correspondence to: Mr Crowther, Department of Orthopaedics, University of Bristol, Bristol Royal Infirmary, Upper Maudlin Street, Bristol BS2 8HW, UK; maacrowther@hotmail.com

Accepted 20 July 2004

\section{REFERENCES}

1 Brogle PJ, Eswar S. Propagation of a patellar stress fracture in a basketball player. Am J Orthop 1997;26:782-4

2 Mata SG, Grande MM, Ovejero AH. Transverse stress fracture of the patella. Clin J Sport Med 1996;6:259-61.

3 Teitz CC, Harrington RM. Patellar stress fracture. Am J Sports Med 1992;20:761-5.

4 Boden BP, Osbahr DC. High-risk stress fractures: evaluation and treatment. $J$ Am Acad Orthop Surg 2000;8:344-53.

5 Mayers LB, Khabie V, Castorina R, et al. Acute transverse patellar fracture associated with weightliffing. Am J Sports Med 2001;29:232-3. 